\title{
An open, multi-centre, phase II clinical trial to evaluate the efficacy and safety of paclitaxel, UFT, and leucovorin in patients with advanced gastric cancer
}

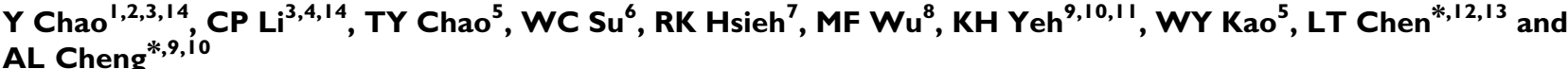

'Cancer Center, Taipei Veterans General Hospital, Taipei, Taiwan; ${ }^{2}$ Central Clinic Hospital, Taipei, Taiwan; ${ }^{3}$ National Yang-Ming University School of Medicine, Taipei, Taiwan; ${ }^{4}$ Division of Gastroenterology, Department of Medicine, Taipei Veterans General Hospital, Taipei, Taiwan; ${ }^{5}$ Division of Hematology and Oncology, Tri-Service General Hospital, National Defense Medical Center, Taipei, Taiwan; ${ }^{6}$ Department of Internal Medicine, National Cheng Kung University Hospital, Tainan, Taiwan; ' Division of Hematology and Oncology, Department of Internal Medicine, Mackay Memorial Hospital, Taipei, Taiwan; ${ }^{8}$ Department of Internal Medicine, Chung Shan Medical University Hospital, Taichung, Taiwan; ${ }^{9}$ Department of Oncology, National Taiwan University Hospital, Taipei, Taiwan; ${ }^{10}$ Cancer Research Center, National Taiwan University College of Medicine, Taipei, Taiwan; "Division of Oncology and Hematology, Department of Intemal Medicine, Far Eastern Memorial Hospital, Taipei, Taiwan; ${ }^{2}$ Institute of Cancer Research, National Health Research Institutes, Taipei, Taiwan; ${ }^{13}$ Department of Internal Medicine, Kaohsiung Medical University Hospital, Kaohsiung, Taiwan

The aim of the study was to evaluate the response rate and safety of weekly paclitaxel $\left(\right.$ Taxol $\left.{ }^{\circledR}\right)$ combination chemotherapy with UFT (tegafur, an oral 5-fluorouracil prodrug, and uracil at a 1:4 molar ratio) and leucovorin (LV) in patients with advanced gastric cancer. Patients with histologically confirmed, locally advanced or recurrent/metastatic gastric cancer were studied. Paclitaxel I-h infusion at a dose of $100 \mathrm{mg} \mathrm{m}^{-2}$ on days I and 8 and oral UFT $300 \mathrm{mg} \mathrm{m}^{-2}$ day $^{-1}$ plus LV $90 \mathrm{mg} \mathrm{day}^{-1}$ were given starting from day I for 14 days, followed by a 7-day period without treatment. Treatment was repeated every 21 days. From February 2003 to October 2004, 55 patients were enrolled. The median age was 62 years (range: $32-82$ ). Among the 48 patients evaluated for tumour response, two achieved a complete response and 22 a partial response, with an overall response rate of 50\% (95\% confidence interval: 35-65\%). All 55 patients were evaluated for survival and toxicities. Median time to progression and overall survival were 4.4 and 9.8 months, respectively. Major grade 3-4 toxicities were neutropenia in 25 patients (45\%) and diarrhoea in eight patients (15\%). Although treatment was discontinued owing to treatment-related toxicities in nine patients (16\%), there was no treatment-related mortality. Weekly paclitaxel plus oral UFT/LV is effective, convenient, and well tolerated in treating patients with advanced gastric cancer.

British Journal of Cancer (2006) 95, I59- 163. doi: I0.1038/sj.bjc.6603225 www.bjcancer.com

Published online 27 June 2006

(c) 2006 Cancer Research UK

Keywords: paclitaxel; UFT; leucovorin; gastric cancer

Gastric cancer is the second leading cause of cancer death worldwide (Roder, 2002). The prognosis is generally poor, with an overall 5 -year survival of approximately $20 \%$ in most countries. The median survival time of patients who presented with advanced or metastatic diseases and received best supportive care was only 3-5 months (Verdecchia et al, 2003). For decades, gastric cancer has been considered as a chemo-resistant tumour. With the introduction of novel drug administration schedules and the

*Correspondence: Dr LT Chen, Institute of Cancer Research, National Health Research Institutes, Taipei Veterans General Hospital, Ward 191, No. 20I, Sec. 2, Shih-Pai Road, Taipei I12, Taiwan;

E-mail: leochen@nhri.org.tw or AL Cheng, Department of Internal Medicine and Department of Oncology, National Taiwan University Hospital, No. 2, Chung-Shan S Rd, Taipei 100, Taiwan;

E-mail: andrew@ha.mc.ntu.edu.tw

${ }^{14}$ These authors contributed equally to this work.

Received 6 February 2006; revised 17 May 2006; accepted 19 May 2006; published online 27 June 2006 emergence of new chemotherapeutic agents, modern systemic chemotherapy could achieve an objective response rate of $30-60 \%$ in advanced gastric cancers (Schoffski, 2002). The overall survival (OS) also improved to a range between 9 and 11 months; however, significant treatment-related toxicities were usually inevitable (Ohtsu, 2005). New treatments with better therapeutic index are needed to improve the outcome.

Weekly infusion of high-dose 5-fluorouracil (5-FU)/leucovorin (LV), the HDFL regimen, is an effective chemotherapy regimen for advanced gastric cancer, with a response rate of $33-48 \%$ and a median survival of 9-10 months, and the haematological toxicities are minimal (Hsu et al, 1997; Lin et al, 1999). It can be safely applied even to patients with a poor general condition (Yeh and Cheng, 1998; Lee et al, 2002). For its efficacy and low-toxicity profile, weekly HDFL per se and HDFL-based new combination regimens have been extensively evaluated for the treatment of patients with advanced gastric cancer in Taiwan (Lin et al, 2001; Chen et al, 2002; Chao et al, 2004; Yeh et al, 2005). However, the implantation of a central venous catheter is inevitable for these treatments. 
UFT, a combination of tegafur (a prodrug of 5-FU) and uracil in a 1:4 molar ratio, is a second generation of oral fluoropyrimidine. Uracil serves as a competitive antagonist for dihydropyrimidine dehydrogenase, the major catabolic enzyme of 5-FU, to enhance the concentration and half-life of 5-FU in the circulation. Pharmacokinetically, orally administered UFT simulates the continuously intravenous infusion of 5-FU (Meropol et al, 1996; Ho et al, 1998). On a 28-day schedule, oral UFT monotherapy could achieve a $20 \%$ response rate in advanced gastric cancer without significant myelosuppression, diarrhoea, and stomatitis (Anderson and Lokich, 1992; Takiuchi and Ajani, 1998).

Paclitaxel, an antimitotic agent that stabilises microtubules, exhibits in vitro activity against gastric cancer cells (Caplow et al, 1994). Paclitaxel monotherapy, at a dose range of $200-250 \mathrm{mg} \mathrm{m}^{-2}$ given as either a 24 - or a 3-h infusion every 3 weeks, could achieve a tumour response rate of $17-21 \%$ and a median response duration of 6.5 months in advanced gastric cancer (Ajani et al, 1998; Ohtsu et al, 1998; Yamada et al, 2001; Schoffski, 2002; Ohtsu, 2005). The non-overlapping toxicity profile of paclitaxel and infusional 5-FU, and the observation of a schedule-dependent synergism between paclitaxel and 5-FU in human gastric cancer cells (Yeh et al, 1998), rationalise such a combination to be evaluated in treating advanced gastric cancers.

We conducted a phase II study of combination chemotherapy of paclitaxel, UFT, and LV to determine the response rate and toxicity profile of this combination in patients with advanced gastric cancer.

\section{PATIENTS AND METHODS}

\section{Patients}

Eligibility criteria of the patients included (1) pathologically confirmed, locally advanced (unresectable), recurrent or metastatic gastric cancer, (2) measurable disease by imaging studies, (3) no prior chemotherapy except postoperative adjuvant chemotherapy that had been administrated more than 12 months before entering into the study, (4) an ECOG (Eastern Cooperative Oncology Group) performance status $\leqslant 2$, (5) age greater than 18 years old, and (6) adequate hepatic, renal, and bone marrow functions.

Exclusion criteria included (1) pre-existing peripheral neuropathy, (2) pregnancy, breastfeeding, or women of childbearing potential without adequate contraception, (3) a concurrent or prior malignancy, (4) central nervous system metastases, (5) active infection, and (6) concurrent treatments that might interfere with study evaluation. This study was approved by the ethics committee of all participating institutes and signed informed consent was obtained from all patients.

\section{Study design}

This was a prospective, multi-centre, phase II clinical trial. The primary objective was to evaluate the response rate of weekly paclitaxel combination chemotherapy with UFT and LV in patients with advanced gastric cancer. The secondary objective was to determine the time to progression (TTP), OS, and safety.

\section{Chemotherapy protocol}

Paclitaxel (Taxol ${ }^{\mathbb{R}}$, Bristol-Myers Squibb, Princeton, NJ, USA) was administered as a 1-h continuous intravenous infusion at a dose of $100 \mathrm{mg} \mathrm{m}^{-2}$ on days 1 and 8 . To reduce the risk of hypersensitivity reactions to paclitaxel, all patients were premedicated with $10 \mathrm{mg}$ of dexamethasone, $300 \mathrm{mg}$ of cimetidine, and $50 \mathrm{mg}$ of diphenhydramine intravenous infusion $30 \mathrm{~min}$ before chemotherapy. UFT $^{\mathbb{R}} \quad$ (Taiho Pharmaceutical Co. Ltd., Tokyo, Japan) $300 \mathrm{mg} \mathrm{m}^{-2}$ day $^{-1}$ and LV (Leucovorin ${ }^{\mathbb{R}}$, Wyeth Farma, SA Madrid, Spain) $90 \mathrm{mg} \mathrm{day}^{-1}$ were administered orally starting from day 1 and continuing for 14 days, followed by a 7-day period off treatment. The total daily dose of UFT was determined and rounded to the nearest $100 \mathrm{mg}$ and divided into three doses given $8 \mathrm{~h}$ apart. If the total number of tablets could not be evenly divided, the highest dose was given in the morning and lower doses in the afternoon or evening. Cycles were repeated every 3 weeks or upon recovery from toxicities to baseline or grade 1 (except alopecia and anaemia).

\section{Dose modification}

Chemotherapy doses may be reduced or treatment may be delayed for no more than 2 weeks to allow for patients' recovery. The dose of each study drug was reduced stepwise. Level 1 was paclitaxel $80 \mathrm{mg} \mathrm{m}^{-2}$, UFT $250 \mathrm{mg} \mathrm{m}^{-2}$, and LV $90 \mathrm{mg} \mathrm{day}^{-1}$, and level 2 was paclitaxel $60 \mathrm{mg} \mathrm{m}^{-2}$, UFT $200 \mathrm{mg} \mathrm{m}^{-2}$, and $\mathrm{LV} 90 \mathrm{mg} \mathrm{day}^{-1}$. A maximum of two dose-level reductions were allowed per patient. Any patient who required a reduction of dose to lower than level 2 must be excluded from the treatment protocol. Dose modification was based on haematological toxicity and on non-haematological toxicity. At haematological nadir, if the neutrophil count was $<500 \mathrm{~mm}^{-3}$ or platelets $<25000 \mathrm{~mm}^{-3}$, the next dose was reduced by one level. If a haematology test within $72 \mathrm{~h}$ before the next cycle indicated that the neutrophil count was $<1000 \mathrm{~mm}^{-3}$ or the platelet count was $<100000 \mathrm{~mm}^{-3}$, the next cycle should be delayed until haematological recovery. Haematology test should be performed every week to identify the haematological recovery. If recovery was not achieved within 2 weeks from the scheduled date of next cycle, the patient must be taken off study protocol. If haematological recovery was not achieved before day 8 from the scheduled date, then paclitaxel administration was skipped on day 8 .

Non-haematological toxicities were graded. Paclitaxel administration was resumed at the same dose level during a cycle upon recovery from toxicities to the baseline or grade 1 (except alopecia and anaemia). If recovery from non-haematological toxicity was not achieved before day 8 from the scheduled date, the paclitaxel dose was skipped on day 8 and the next dose was reduced by one level. For a toxicity greater than grade 2, UFT and LV administration was interrupted. When the toxicity subsided to baseline or less than grade 1, administration was resumed. Treatment was not continued after treatment cycle day 14, regardless of the number of days the drug has been interrupted.

\section{Evaluation of efficacy and toxicities}

Evaluations before chemotherapy included medical history taking, physical examination, complete blood count, blood chemistry, chest X-ray, and computed tomography (CT) scan and/or magnetic resonance imaging (MRI) of abdomen. After starting protocol treatment, a complete blood count was conducted weekly and blood chemistry every 3 weeks. Detailed history, physical examinations, and treatment-related toxicities were recorded weekly. Tumour size was measured by imaging studies (CT and/ or MRI) every 6 weeks. Tumour response was evaluated according to the World Health Organization criteria (Miller et al, 1981). All subjects with tumour responses (complete response (CR) and partial response (PR)) underwent a confirmatory scan 4 weeks following the initial documentation. Toxicities were graded according to the NCI-Common Toxicity Criteria (version 2) (Trotti et al, 2000).

\section{Statistical analyses}

The Simon optimal two-stage design was used (Simon, 1988). The response rate of interest were $P_{0}=30 \%$ and $P_{1}=50 \%$. If objective tumour responses were observed in more than five of 15 evaluable patients in the first stage, an additional 31 evaluable patients would 
be enrolled in the second stage. If there were more than 19 responders at the end of the second stage, this treatment would be considered as effective and deserving further investigation, with an $\alpha$-value of 0.05 and a $\beta$-value of 0.10 . Time to progression was defined as the duration from the date of starting protocol treatment to the date of documented disease progression or death by any cause. Overall survival was defined as the duration from the date of starting protocol treatment to the date of death. Survival was estimated by Kaplan-Meier analyses.

\section{RESULTS}

\section{Patients and treatment}

Between February 2003 and October 2004, 55 patients were enrolled into the study from six medical centres. The major clinicopathologic characteristics of patients are listed in Table 1. The median age of the patients was 62 years (range: $32-82$ ). A total of 343 (median: 6; range: 1-21) cycles of chemotherapy were given. Median relative dose intensity was $98 \%$ (range: $70-100 \%$ ) for paclitaxel, $88 \%$ (range: $7-100 \%$ ) for UFT, and $88 \%$ (range: $7-$ $100 \%$ ) for LV. In total, $96 \%$ of the patients received more than $80 \%$ of the intended doses of paclitaxel, and $56 \%$ received more than $80 \%$ of the intended doses of UFT and LV.

\section{Efficacy}

Seven patients were not evaluable for tumour responses: four patients failed to return to the clinic for tumour measurements, two patients were found to have no measurable tumours later, and one patient refused chemotherapy. Among the 48 evaluable patients, the best tumour response was CR in two patients, PR in 22 patients, stable disease in 20 patients, and progressive disease in four patients. The overall response rate was $50 \%$ (24 out of 48

Table I Clinicopathologic features of the patients

\begin{tabular}{lc}
\hline & Patient number (\%) \\
\hline Total patients & 55 \\
Age (years), median (range) & $62(32-82)$ \\
Sex: male/female & $33 / 22$ \\
ECOG performance & \\
0 & $4(7)$ \\
I & $45(82)$ \\
2 & $6(11)$ \\
Treatments for primary tumour & \\
No prior therapy & \\
Surgery only & $28(51)$ \\
Surgery+adjuvant chemotherapy & $24(44)$ \\
Radiotherapy & $2(4)$ \\
& $1(2)$ \\
Disease status & \\
Locally advanced & \\
Recurrence/metastasis & $3(5)$ \\
Disease sites & $52(95)$ \\
Liver & \\
Lymph nodes & \\
Peritoneum & $11(20)$ \\
Gastrointestinal tract & $29(53)$ \\
Bone & $24(44)$ \\
Lung & $12(22)$ \\
Others & $14(25)$ \\
\hline
\end{tabular}

ECOG = Eastern Cooperative Oncology Group. patients, 95\% confidence interval (CI): 35-65\%). The response rates for patients with $\geqslant 80$ and $<80 \%$ of scheduled UFT dose intensity were $57 \%$ ( 17 out of 30 patients) and $39 \%$ ( 8 out of 18 patients), respectively $(P=0.3715)$. The median time to tumour response was 3 (range: $2.1-4.4)$ months. The median duration of tumour response was 5.9 (range: $0.4-12.6$ ) months.

On an intention-to-treat analysis, the median follow-up time for the 55 enrolled patients was 11.7 (range: 1.0-30.0) months. The median TTP and OS were 4.4 (95\% CI: 4.0-6.5) months and 9.8 (95\% CI: 8.6-10.7) months, respectively. The Kaplan-Meier estimated TTP and OS curves are shown in Figures 1 and 2.

\section{Toxicity}

All 55 patients were evaluated for toxicities (Table 2). The most common toxicity was neutropenia, with grade 3-4 neutropenia observed in $45 \%$ of the 55 patients evaluable for toxicity. One patient developed neutropenic fever and recovered with appropriate therapy. Grade 3 sensory neuropathy developed in four (7\%) patients after a median of five cycles of treatment that invariably improved with discontinuation of chemotherapy. Grade 3-4 diarrhoea was observed in $15 \%$ of the patients. Alopecia developed in 40 patients $(73 \%)$. Grade 3 sinus tachycardia was noted in $5 \%$ of the patients. Among them, two occurred during infusion of the paclitaxel and the other was not related to paclitaxel. All recovered spontaneously. A total of $32(58 \%)$ patients had a dose delay during treatment. Dose modification was required in $22(40 \%)$

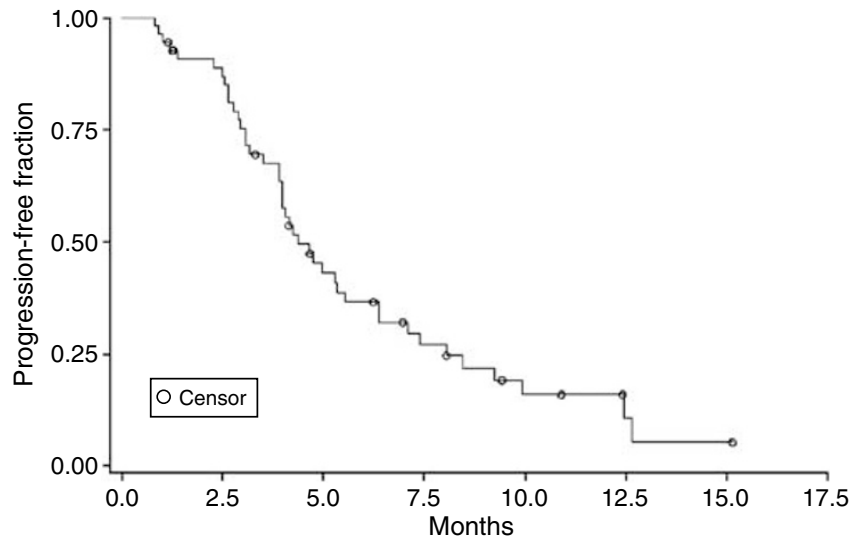

Figure I Time to progression of the 55 patients.

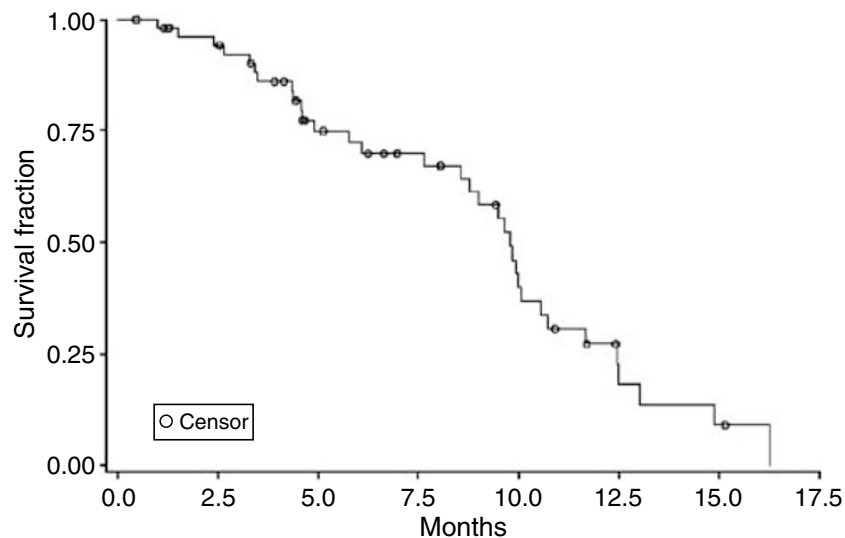

Figure 2 Overall survival of the 55 patients. 
Table 2 Percentage toxicity of the paclitaxel, UFT, and LV regimen

\begin{tabular}{|c|c|c|c|c|}
\hline \multirow[b]{3}{*}{ Toxicity } & \multicolumn{2}{|c|}{ Patients $(n=55)$} & \multicolumn{2}{|c|}{ Cycles $(n=343)$} \\
\hline & Grade & Grade & Grade & Grade \\
\hline & $1-2$ & $3-4$ & $1-2$ & $3-4$ \\
\hline \multicolumn{5}{|l|}{ Haematological } \\
\hline Neutropenia & $25^{a}$ & 45 & $36^{b}$ & 22 \\
\hline Leucopenia & 55 & 18 & 52 & 6 \\
\hline Thrombocytopenia & 2 & 2 & I & 0.6 \\
\hline Febrile neutropenia & 4 & 2 & 0.6 & 0.3 \\
\hline Anaemia & 42 & 9 & 28 & 2 \\
\hline \multicolumn{5}{|l|}{ Gastrointestinal } \\
\hline Nausea & 27 & 0 & 14 & 0 \\
\hline Vomiting & 20 & 0 & 6 & 0 \\
\hline Diarrhoea & 33 & 15 & 18 & 4 \\
\hline Stomatitis & 5 & 0 & I & 0 \\
\hline Anorexia & 35 & 2 & 20 & 0.3 \\
\hline Weight loss & 5 & 0 & 4 & 0 \\
\hline Hypoaesthesia & 45 & 7 & 46 & 0 \\
\hline \multicolumn{5}{|l|}{ Others } \\
\hline Cardiac & 7 & 5 & । & । \\
\hline Fever & 2 & 0 & 0.3 & 0 \\
\hline Alopecia & 73 & 0 & 74 & 0 \\
\hline
\end{tabular}

$\mathrm{LV}=$ leucovorin. ${ }^{\mathrm{a}} \mathrm{All}$ numbers are percentage of the 55 patients. ${ }^{\mathrm{b}} \mathrm{All}$ numbers are percentage of the 343 cycles given.

patients. Treatment-related toxicity resulted in treatment discontinuation in nine $(16 \%)$ patients, which were associated with paclitaxel-related neuropathy in four patients, a delayed recovery from neutropenia in four patients, and suspicious myocardial ischaemia (chest pain) in one patient. There was no treatmentrelated mortality.

\section{DISCUSSION}

In this study, we showed that weekly $100 \mathrm{mg} \mathrm{m}^{-2}$ of paclitaxel plus daily oral UFT/LV for 2 weeks every 3 weeks is an active combination chemotherapy regimen for patients with advanced gastric cancer. The $50 \%$ overall response rate of the evaluable patients ( $44 \%$ on intention-to-treat analyses), a median survival of 9.8 months, and $45 \%$ of grade $3-4$ neutropenia were compatible with those achieved with topoisomerase I inhibitor-, taxane-, or third generation of oral fluoropyrimidine (i.e. TS-1 and capecitabine)-based doublet chemotherapy in phase II trials (Schoffski, 2002; Ohtsu, 2005), in which the objective tumour response rate and median survival ranged from 40 to $76 \%$ and from 9 to 12.5 months, respectively. The median survival of this study was also comparable with those achieved in previous HDFL-based studies in our institutes (Hsu et al, 1997; Chen et al, 2002; Chao et al, 2004), and not inferior to the 6.1-12.0 months of median survival of phase II or III studies of current 'reference' regimens for advanced gastric cancers, that is, ECF, FAMTX, FAMTX, ELF, FUP, FOLFOX6, and DCF (Webb et al, 1997; De Vivo et al, 2000; Vanhoefer et al, 2000; Louvet et al, 2002; Ajani et al, 2005; Ohtsu, 2005).

Recently, the superior therapeutic index of weekly paclitaxel compared to the triweekly schedule has been demonstrated in gastric cancer patients (Ohtsu et al, 1998; Yamada et al, 2001; Kakeji et al, 2005). With a comparable tumour response rate (22$23 \%)$ for advanced gastric cancer, the grade 3-4 neutropenia of weekly paclitaxel $\left(80 \mathrm{mg} \mathrm{m}^{-2}\right.$, on days 1,8 , and 15 every 4 weeks) and of triweekly paclitaxel $\left(200-210 \mathrm{mg} \mathrm{m}^{-2}\right.$, on day 1 every 3 weeks) were 20 and $37-67 \%$, respectively. Weekly paclitaxel plus either infusional 5-FU or oral fluoropyrimidine have become popular investigational combinations for advanced gastric cancers (Ninomiya et al, 2005; Takeyoshi et al, 2005; Ueda et al, 2005; Yeh et al, 2005). Among these phase II studies, including the current one, the tumour response rate was quite consistent, ranging from 35 to $50 \%$ in chemo-naïve gastric cancer patients (Ninomiya et al, 2005; Takeyoshi et al, 2005; Yeh et al, 2005). The efficacy parameters (objective response, TTP, and OS) and toxicity profiles (esp. grade 3-4 neutropenia) of current regimen were also nearly identical to those observed in the previous paclitaxel plus infusional HDFL trial performed by Yeh et al (2005). The advantage of this oral approach is the alleviation of the requirement for and the cost of central venous catheter implantation and the associated inconvenience of infusion pump, which in turn might improve the quality of life of treated patients. Our results suggest that, while in combination with weekly paclitaxel, daily administration of oral UFT/LV might achieve a similar therapeutic index and ultimately replace intermittent infusional $5-\mathrm{FU} / \mathrm{LV}$ treatment for advanced gastric cancer.

Recently, Takeyoshi et al (2005) showed that paclitaxel $80 \mathrm{mg} \mathrm{m}^{-2}$ intravenously on days 1,8 , and 15 every 4 weeks and doxifluridine ( 5 -dexoy-5-fluorouridine, an intermediate metabolite of capecitabine) $533 \mathrm{mg} \mathrm{m}^{-2}$ orally on days $1-5$ per week could achieve a tumour response rate of $46 \%$ with only $12 \%$ of grade $3-4$ leucopenia in chemo-naïve patients. Although the results (including the unexpected low incidence of myelosuppression) have to be verified by a large-scale clinical trial, the 5-days-on/2-days-off oral fluoropyrimidine schedule is of interest. A recent pharmacological study on surgical specimens from colorectal cancer patients who had received preoperative 5-days (weekday)-on/2-days (weekend)off UFT treatment showed maintenance of a relatively high level of intratumoral concentration of 5-FU at $48 \mathrm{~h}$ after the last dose of UFT (Sadahiro et al, 2001). The schedule was considered to reduce the incidence of adverse events and to improve treatment compliance. Can the modification of UFT administration schedule further improve the therapeutic index of currently weekly paclitaxel plus UFT/LV regimen deserves further exploration.

Grade 3 sinus tachycardia was noted in $5 \%$ of the patients. Asymptomatic cardiac disturbances have been reported during paclitaxel infusion (Kamineni et al, 2003). Hypersensitivity to paclitaxel or coexistence of underlying diseases may be the aetiologies. The exact cause was unknown. Caution should be exercised in patients with underlying cardiac diseases and more prospective studies are needed to assess its cardiotoxicities.

Nowadays, several novel targeted therapeutic agents such as inhibitors of epidermal growth factor receptor or of vascular endothelial growth factor, when used in combination with chemotherapy, have shown promising activity against gastrointestinal cancers (Chong and Cunningham, 2005). The lowtoxicity profiles of such agents also provide an excellent chance to improve the therapeutic index of this active and convenient regimen for advanced gastric cancer patients.

In conclusion, the combination of weekly paclitaxel and oral UFT/LV is an active, outpatient-based chemotherapy regimen with acceptable toxicities. However, for the palliative nature of systemic chemotherapy in recurrent or metastatic gastric cancer, strategies to improve the therapeutic index of the current regimen, that is, modification of drug administration schedule and/or in combination with novel biological targeted agents, should also be further explored.

\section{ACKNOWLEDGEMENTS}

This study was supported by Bristol-Myers Squibb Company. 


\section{REFERENCES}

Ajani JA, Fairweather J, Dumas P, Patt YZ, Pazdur R, Mansfield PF (1998) Phase II study of Taxol in patients with advanced gastric carcinoma. Cancer J Sci Am 4: 269-274

Ajani JA, Fodor MB, Tjulandin SA, Moiseyenko VM, Chao Y, Cabral Filho S, Majlis A, Assadourian S, Van Cutsem E (2005) Phase II multiinstitutional randomized trial of docetaxel plus cisplatin with or without fluorouracil in patients with untreated, advanced gastric, or gastroesophageal adenocarcinoma. J Clin Oncol 23: 5660-5667

Anderson N, Lokich J (1992) Controversial issues in 5-fluorouracil infusion use. Dose intensity, treatment duration, and cost comparisons. Cancer 70(Suppl): $998-1002$

Caplow M, Shanks J, Ruhlen R (1994) How taxol modulates microtubule disassembly. J Biol Chem 269: 23399-23402

Chao Y, Yeh KH, Chang CJ, Chen LT, Chao TY, Wu MF, Chang CS, Chang JY, Chung CY, Kao WY, Hsieh RK, Cheng AL (2004) Phase II study of oxaliplatin and weekly 24-hour infusion of high-dose 5-fluorouracil and folinic acid in the treatment of advanced gastric cancer. $\mathrm{Br} J$ Cancer 90: $453-458$

Chen LT, Liu TW, Wu CW, Chung TR, Shiah HS, Jan CM, Liu JM, WhangPeng J, Chang JY (2002) A phase I study of weekly docetaxel, 24-hour infusion of high-dose fluorouracil/leucovorin and cisplatin in patients with advanced gastric cancer. Oncology 63: 239-247

Chong G, Cunningham D (2005) Gastrointestinal cancer: recent developments in medical oncology. Eur J Surg Oncol 31: 453-460

De Vivo R, Pignata S, Palaia R, Parisi V, Daniele B (2000) The role of chemotherapy in the management of gastric cancer. J Clin Gastroenterol 30: $364-371$

Ho DH, Pazdur R, Covington W, Brown N, Huo YY, Lassere Y, Kuritani J (1998) Comparison of 5-fluorouracil pharmacokinetics in patients receiving continuous 5-fluorouracil infusion and oral uracil plus N1-(2'-tetrahydrofuryl)-5-fluorouracil. Clin Cancer Res 4: 2085-2088

Hsu CH, Yeh KH, Chen LT, Liu JM, Jan CM, Lin JT, Chen YC, Cheng AL (1997) Weekly 24-hour infusion of high-dose 5-fluorouracil and leucovorin in the treatment of advanced gastric cancers. Oncology 54: $275-280$

Kakeji Y, Emi Y, Baba H, Tokunaga E, Oki E, Yamamoto M, Ikebe M, Maehara Y, Hashizume M (2005) Efficacy of weekly paclitaxel in patients with advanced gastric cancer: phase II study in Japan. 2005 Gastrointestinal Cancers Symposium, abstract 53

Kamineni P, Prakasa K, Hasan SP, Akula R, Dawkins F (2003) Cardiotoxicities of paclitaxel in African Americans. I Natl Med Assoc 95: $977-981$

Lee KD, Liu TW, Wu CW, Tiu CM, Liu JM, Chung TR, Chang JY, WhangPeng J, Chen LT (2002) Non-surgical treatment for afferent loop syndrome in recurrent gastric cancer complicated by peritoneal carcinomatosis: percutaneous transhepatic duodenal drainage followed by 24-hour infusion of high-dose fluorouracil and leucovorin. Ann Oncol 13: $1151-1155$

Lin YC, Chen JS, Wang CH, Wang HM, Chang HK, Liaul CT, Yang TS, Liaw CC, Liu HE (2001) Weekly high-dose 5-fluorouracil (5-FU), leucovorin (LV) and bimonthly cisplatin in patients with advanced gastric cancer. Jpn J Clin Oncol 31: 605-609

Lin YC, Liu HE, Wang CH, Wang HM, Yang TS, Liau CT, Chen JS (1999) Clinical benefit and response in patients with gastric cancer to weekly 24hour infusion of high-dose 5-fluorouracil (5-FU) and leucovorin (LV). Anticancer Res 19: 5615-5620

Louvet C, Andre T, Tigaud JM, Gamelin E, Douillard JY, Brunet R, Francois E, Jacob JH, Levoir D, Taamma A, Rougier P, Cvitkovic E, de Gramont A (2002) Phase II study of oxaliplatin, fluorouracil, and folinic acid in locally advanced or metastatic gastric cancer patients. J Clin Oncol 20: $4543-4548$

Meropol NJ, Rustum YM, Petrelli NJ, Rodriguez-Bigas M, Frank C, Ho DH, Kurowski M, Creaven PJ (1996) A phase I and pharmacokinetic study of oral uracil, ftorafur, and leucovorin in patients with advanced cancer. Cancer Chemother Pharmacol 37: $581-586$

Miller AB, Hoogstraten B, Staquet M, Winkler A (1981) Reporting results of cancer treatment. Cancer 47: 207-214

Ninomiya M, Kondo K, Kojima H, Matsuo K, Hirabayashi N, Nakamura S, Kawamura S, Kobayashi M, Konno H, Sakamoto J (2005) Phase II study of weekly paclitaxel plus 5-fluorouracil in patients with unresectable advanced or recurrent gastric cancer. 2005 Proceedings of the American Society of Clinical Oncology, abstract 4075

Ohtsu A (2005) Current status and future prospects of chemotherapy for metastatic gastric cancer: a review. Gastric Cancer 8: $95-102$

Ohtsu A, Boku N, Tamura F, Muro K, Shimada Y, Saigenji K, Akazawa S, Kitajima M, Kanamaru R, Taguchi T (1998) An early phase II study of a 3-hour infusion of paclitaxel for advanced gastric cancer. Am J Clin Oncol 21: $416-419$

Roder DM (2002) The epidemiology of gastric cancer. Gastric Cancer 5(Suppl 1): $5-11$

Sadahiro S, Suzuki T, Kameya T, Iwase H, Tajima T, Makuuchi H (2001) A pharmacological study of the weekday-on/weekend-off oral UFT schedule in colorectal cancer patients. Cancer Chemother Pharmacol 47: $457-460$

Schoffski P (2002) New drugs for treatment of gastric cancer. Ann Oncol 13(Suppl 4): $13-22$

Simon R (1988) Optimal two-stage design for phase II clinical trials. Control Clin Trials 10: $1-10$

Takeyoshi I, Makita F, Tanahashi Y, Iwazaki S, Nakamura S, Ogawa T, Ishikawa H, Ohya T, Kawate S, Morishita Y (2005) A phase II study of weekly paclitaxel and doxifluridine (an intermediate metabolite of capecitabine) combination chemotherapy for advanced/recurrent gastric cancer. 2005 Proceedings of the American Society of Clinical Oncology, abstract 4082

Takiuchi H, Ajani JA (1998) Uracil-tegafur in gastric carcinoma: a comprehensive review. J Clin Oncol 16: 2877-2885

Trotti A, Byhardt R, Stetz J, Gwede C, Corn B, Fu K, Gunderson L, McCormick B, Morrisintegral M, Rich T, Shipley W, Curran W (2000) Common toxicity criteria: version 2.0. An improved reference for grading the acute effects of cancer treatment: impact on radiotherapy. Int J Radiat Oncol Biol Phys 47: 13-47

Ueda Y, Yamagishi H, Ichikawa D, Morii J, Koizumi K, Kakihara N, Shimotsuma M, Takenaka A, Yamashita T, Kurioka H, Nishiyama M, Morita S, Nakamura K, Sakamoto J (2005) Phase I study of a combination of s-1 and weekly paclitaxel in patients with advanced or recurrent gastric cancer. Oncology 69: $261-268$

Vanhoefer U, Rougier P, Wilke H, Ducreux MP, Lacave AJ, Van Cutsem E, Planker M, Santos JG, Piedbois P, Paillot B, Bodenstein H, Schmoll HJ, Bleiberg H, Nordlinger B, Couvreur ML, Baron B, Wils JA (2000) Final results of a randomized phase III trial of sequential high-dose methotrexate, fluorouracil, and doxorubicin versus etoposide, leucovorin, and fluorouracil versus infusional fluorouracil and cisplatin in advanced gastric cancer: a trial of the European Organization for Research and Treatment of Cancer Gastrointestinal Tract Cancer Cooperative Group. J Clin Oncol 18: 2648-2657

Verdecchia A, Mariotto A, Gatta G, Bustamante-Teixeira MT, Ajiki W (2003) Comparison of stomach cancer incidence and survival in four continents. Eur J Cancer 39: 1603-1609

Webb A, Cunningham D, Scarffe JH, Harper P, Norman A, Joffe JK, Hughes M, Mansi J, Findlay M, Hill A, Oates J, Nicolson M, Hickish T, O’Brien M, Iveson T, Watson M, Underhill C, Wardley A, Meehan M (1997) Randomized trial comparing epirubicin, cisplatin, and fluorouracil versus fluorouracil, doxorubicin, and methotrexate in advanced esophagogastric cancer. J Clin Oncol 15: $261-267$

Yamada Y, Shirao K, Ohtsu A, Boku N, Hyodo I, Saitoh H, Miyata Y, Taguchi T (2001) Phase II trial of paclitaxel by three-hour infusion for advanced gastric cancer with short premedication for prophylaxis against paclitaxel-associated hypersensitivity reactions. Ann Oncol 12: $1133-1137$

Yeh KH, Cheng AL (1998) Gastric cancer associated with acute disseminated intravascular coagulation: successful initial treatment with weekly 24-hour infusion of high-dose 5-fluorouracil and leucovorin. $\mathrm{Br} J$ Haematol 100: 769-772

Yeh KH, Cheng AL, Lin JF, Ma IF, Hsu CH, Lu YS, Chen YC (1998) Sequence-dependent synergism of paclitaxel and 5-fluorouracil in the treatment of gastric cancer: in vitro and pilot clinical studies. Ann Oncol 9(Suppl 4): 47 (abstract 225)

Yeh KH, Lu YS, Hsu CH, Lin JF, Hsu C, Kuo SH, Li SJ, Cheng AL (2005) Phase II study of weekly paclitaxel and 24-hour infusion of high-dose 5 -fluorouracil and leucovorin in the treatment of recurrent or metastatic gastric cancer. Oncology 69: 88-95 